\title{
253.
}

\section{SUR LA COURBE PARALLÈLE À L'ELLIPSE.}

[From the Annali di Matematica pura ed applicata (Tortolini), tom. III. (1860), pp. 311-316.]

IL fut remarqué par Cauchy (Comptes Rendus, tom. xiII. [1841], p. 1063) que l'équation de cette courbe pourrait se trouver en éliminant $\theta$ entre les équations

$$
\frac{a^{2} x^{2}}{\left(\theta+a^{2}\right)^{2}}+\frac{b^{2} y^{2}}{\left(\theta+b^{2}\right)^{2}}=1, \quad \frac{\theta^{2} x^{2}}{\left(\theta+a^{2}\right)^{2}}+\frac{\theta^{2} y^{2}}{\left(\theta+b^{2}\right)^{2}}=k^{2},
$$

et cette élimination fut effectuée, et le résultat trouvé sous la forme la plus simple par M. Catalan (Terquem, tom. III. 1844, p. 558); mais pour faire l'élimination de la manière la plus facile je remarque que ces deux équations donnent

$$
\frac{x^{2}}{\theta+a^{2}}+\frac{y^{2}}{\theta+b^{2}}=1+\frac{k^{2}}{\theta}, \quad \frac{x^{2}}{\left(\theta+a^{2}\right)^{2}}+\frac{y^{2}}{\left(\theta+b^{2}\right)^{2}}=\frac{k^{2}}{\theta^{2}},
$$

dont la seconde est la dérivée, par rapport à $\theta$, de la première; or cette première équation est

$$
\left(\theta+k^{2}\right)\left(\theta+a^{2}\right)\left(\theta+b^{2}\right)-x^{2} \theta\left(\theta+b^{2}\right)-y^{2} \theta\left(\theta+a^{2}\right)=0,
$$

et, en égalant à zéro le discriminant de la fonction cubique, on aura l'équation de la courbe; en posant

$$
A=x^{2}+y^{2}-k^{2}-a^{2}-b^{2}, \quad B=b^{2} x^{2}+a^{2} y^{2}-a^{2} k^{2}-b^{2} k^{2}-a^{2} b^{2}, \quad C=a^{2} b^{2} k^{2},
$$

la fonction cubique, multipliée par 3, sera

on aura done

$$
\left(3,-A,-B, 3 C \gamma(\theta, 1)^{3} ;\right.
$$

$$
4\left(A^{2}+3 B\right)\left(B^{2}+3 A C\right)-(A B-9 C)^{2}=0,
$$


ou ce qui est la même chose

$$
A^{2} B^{2}+4 B^{3}+4 A^{3} C+18 A B C-27 C^{2}=0,
$$

c'est-à-dire en substituant les valeurs de $A, B, C$, l'équation de la courbe sera

$$
\begin{aligned}
& \left(x^{2}+y^{2}-k^{2}-a^{2}-b^{2}\right)^{2}\left(b^{2} x^{2}+a^{2} y^{2}-a^{2} k^{2}-b^{2} k^{2}-a^{2} b^{2}\right)^{2} \\
+ & 4\left(b^{2} x^{2}+a^{2} y^{2}-a^{2} k^{2}-b^{2} k^{2}-a^{2} b^{2}\right)^{3} \\
+ & 4 a^{2} b^{2} k^{2}\left(x^{2}+y^{2}-k^{2}-a^{2}-b^{2}\right)^{3} \\
+ & 18 a^{2} b^{2} k^{2}\left(x^{2}+y^{2}-k^{2}-a^{2}-b^{2}\right)\left(b^{2} x^{2}+a^{2} y^{2}-a^{2} k^{2}-b^{2} k^{2}-a^{2} b^{2}\right) \\
- & 27 a^{4} b^{4} k^{4}=0
\end{aligned}
$$

laquelle est en effet l'équation trouvée par M. Catalan.

En écrivant $k=0$ l'équation dévient

$$
0=\left(b^{2} x^{2}+a^{2} y^{2}-a^{2} b^{2}\right)\left[\left(x^{2}+y^{2}\right)^{2}-2\left(a^{2}-b^{2}\right)\left(x^{2}+y^{2}\right)+\left(a^{2}-b^{2}\right)^{2}\right],
$$

ce qui équivaut à l'équation de l'ellipse

$$
b^{2} x^{2}+a^{2} y^{2}-a^{2} b^{2}=0
$$

deux fois répétée, et aux équations

$$
(x \pm a e)^{2}+y^{2}=0
$$

(où comme à l'ordinaire $a^{2} e^{2}=a^{2}-b^{2}$ ) lesquelles appartiennent aux droites menées chacune par un foyer réel et un foyer imaginaire de l'ellipse; ces droites sont aussi les tangentes menées à l'ellipse par les quatre foyers. En effet en prenant sur l'ellipse le point imaginaire dont les coordonnées sont

$$
x=\frac{a}{e}, \quad y=i a\left(\frac{1}{e}-e\right),(i=\sqrt{ }-1)
$$

l'équation du cercle, rayon 0, ayant pour centre le point dont il s'agit, sera

$$
\left(x-\frac{a}{e}\right)^{2}+\left[y-i a\left(\frac{1}{e}-e\right)\right]^{2}=0
$$

ce cercle se réduit donc à deux droites, savoir la droite

$$
x-\frac{a}{e}+i\left[y-i a\left(\frac{1}{e}-e\right)\right]=0,
$$

ou, ce qui est la même chose,

$$
x-a e+i y=0,
$$

laquelle est tangente à l'ellipse, et la droite

$$
\left(x-\frac{a}{e}\right)-i\left[y-i a\left(\frac{1}{e}-e\right)\right]=0,
$$

C. IV. 
ou, ce qui est la même chose,

$$
x-a\left(\frac{2}{e}-e\right)-i y=0
$$

laquelle est la droite menée par le point de contact à l'autre point circulaire à l'infini (celui qui n'est pas situé sur la tangente $x-a e+i y=0$ ). Le cercle, comme courbe composée d'une droite tangente à l'ellipse et d'une autre droite menée par le point de contact, a même avec l'ellipse une intersection à trois points réunis. Ces considérations font voir pourquoi au cas $k=0$ les quatre droites $(x \pm a e)^{2}+y^{2}=0$ font partie de la courbe.

En supposant que $k$ est quelconque, mais que l'on a $a=b$, l'équation devient

$$
a^{4}\left(x^{2}+y^{2}\right)^{2}\left[\left(x^{2}+y^{2}-a^{2}\right)^{2}-2 k^{2}\left(x^{2}+y^{2}+a^{2}\right)+k^{4}\right]=0,
$$

où, en écartant le facteur $\left(x^{2}+y^{2}\right)^{2}$ et le facteur constant $a^{4}$, l'équation se réduit à

c'est-à-dire

$$
\left(x^{2}+y^{2}-a^{2}\right)^{2}-2 k^{2}\left(x^{2}+y^{2}+a^{2}\right)+k^{4}=0,
$$

$$
\left[x^{2}+y^{2}-(a+k)^{2}\right]\left[x^{2}+y^{2}-(a-k)^{2}\right]=0 ;
$$

ainsi la courbe se réduit à l'ensemble des deux droites $\left(x^{2}+y^{2}\right)=0$ (chacune deux fois répétée, et des deux cercles

$$
x^{2}+y^{2}-(a+k)^{2}=0, \quad x^{2}+y^{2}-(a-k)^{2}=0
$$

comme évidemment cela doit être.

Par rapport aux singularités de la courbe, la forme de l'équation montre à premier coup d'œil qu'il y a quatre points doubles à l'infini; savoir les deux points circulaires à l'infini (points où l'infini est rencontré par les droites $x^{2}+y^{2}=0$ ), et les deux points où l'infini est rencontré par les droites $b^{2} x^{2}+a^{2} y^{2}=0$. Il y a de plus deux points doubles sur chacun des axes: en effet en écrivant dans l'équation de la courbe $y=0$ l'équation résultante, toute réduction faite, devient

$$
\left[(x-a)^{2}-k^{2}\right]\left[(x+a)^{2}+k^{2}\right]\left[b^{2} x^{2}-\left(a^{2}-b^{2}\right)\left(b^{2}-k^{2}\right)\right]^{2}=0,
$$

ce qui fait voir qu'il y a sur l'axe de $x$ les deux points doubles dont les coordonnées sont données par l'équation

$$
b^{2} x^{2}-\left(a^{2}-b^{2}\right)\left(b^{2}-k^{2}\right)=0 .
$$

Mais pour parvenir à cette conclusion il convient de considérer que l'axe de $x$ rencontre la courbe dans des points tels que pour chacun la distance normale à l'ellipse est égale à $k$ : il y a quatre distances normales $k$, mesurées le long de l'axe; et quatre distances normales $k$ mesurées à des points situés symétriquement par rapport à l'axe, lesquels se rencontrent deux à deux sur l'axe aux points dont les coordonnées sont $b^{2} x^{2}=\left(a^{2}-b^{2}\right)\left(b^{2}-k^{2}\right)$; c'est là l'origine des deux points doubles sur l'axe de $x$. On doit 
aussi remarquer que les coordonnées des points sur l'ellipse qui correspondent à ces points doubles sont données par les équations

$$
x^{2}=\frac{a^{4}}{b^{2}} \frac{b^{2}-k^{2}}{a^{2}-b^{2}}, \quad y^{2}=\frac{a^{2} k^{2}-b^{4}}{a^{2}-b^{2}}
$$

on voit de là que les points sur l'ellipse qui donnent lieu aux deux points doubles sur l'axe de $x$, ne seront réels à moins que $k>\frac{b^{2}}{a^{3}},<b$; les deux points doubles seront cependant réels pour toute valeur $k>0<b$. J'ajoute que pour les valeurs $k \frac{=}{>},<\frac{b^{2}}{a}$, les deux points doubles seront des points conjugués ou isolés; pour $k=\frac{b^{2}}{a}$, chacun de ces points se réunit à la courbe, et à deux points de rebroussement, mais il n'y a pas de singularité visible dans la forme de la courbe; pour $k>\frac{b^{2}}{a}<b$ il y a deux points doubles avec des branches réelles; et pour $k=b$, ces deux points viennent se réunir au centre de l'ellipse et il y a deux branches ayant pour tangente commune à ce point l'axe de $x$; enfin pour $k>b$, les deux points doubles sur l'axe de $x$ deviennent imaginaires.

Des considérations pareilles s'appliquent aux points doubles sur l'axe de $y$; pour $x=0$ l'équation de la courbe se transforme en

$$
\left[(y-b)^{2}-k^{2}\right]\left[(y+b)^{2}+k^{2}\right]\left[a^{2} y^{2}-\left(a^{2}-b^{2}\right)\left(k^{2}-a^{2}\right)\right]^{2}=0
$$

il y a donc sur l'axe de $y$ deux points doubles dont les coordonnées sont données par

$$
a^{2} y^{2}-\left(a^{2}-b^{2}\right)\left(k^{2}-a^{2}\right)=0
$$

ces points correspondent à des points sur l'ellipse dont les coordonnées sont données par

$$
x^{2}=\frac{a^{4}-b^{2} k^{2}}{a^{2}-b^{2}}, \quad y^{2}=\frac{b^{4}}{a^{2}} \frac{k^{2}-a^{2}}{a^{2}-b^{2}}
$$

les points sur l'ellipse ne seront donc réels à moins que $k>\frac{a^{2}}{\bar{b}}$; les deux points doubles seront cependant réels pour toute valeur $k>a$. Pour $k<a$ les deux points doubles seront imaginaires. Pour $k=a$ le centre est point de réunion de deux points doubles et il $\mathrm{y}$ a deux branches qui ont à ce point pour tangente commune l'axe de $y$ : pour $k=\frac{a^{2}}{b}$, chacun des deux points doubles se réunit à deux points de rebroussement; mais il n'y a pas de singularité visible dans la forme de la courbe; enfin pour $k>\frac{a^{2}}{b}$ les deux points doubles se détachent de la courbe et deviennent des points conjugués ou isolés. En résumé, il y a huit points doubles, savoir quatre points doubles à l'infini, et quatre points doubles sur les deux axes.

Il y a de plus 12 points de rebroussement; ces points sont en effet les centres de courbure aux points de l'ellipse pour lesquels le rayon de courbure est égal à $k$; 20-2 
les douze points seront tous imaginaires à moins qque $k$ n'ait une valeur entre les limites $\frac{b^{2}}{a}, \frac{a^{2}}{b}$ : pour une telle valeur de $k$ les douze points seront 4 réels, et 8 imaginaires. Pour $k=\frac{b^{2}}{a}$ les quatre points réels se réunissent deux à deux aux deux points doubles sur l'axe de $x$; pour $k=\frac{a^{2}}{b}$, ils se réunissent deux à deux aux deux points doubles sur l'axe de $y$; cela s'accorde avec ce qui est dit ci-dessus par rapport aux points doubles. On peut encore trouver que le nombre des points de rebroussement est 12 à moyen des équations

$$
A^{2}+3 B=0, \quad B^{2}+3 A C=0, \quad A B-9 C=0,
$$

lesquelles appartiennent chacune à une courbe du quatrième ordre qui passe par les points de rebroussement; la forme des équations fait voir que ces courbes ont en commun 12 points, et seulement 12 points, d'intersection. C'est là en effet la manière la plus simple de trouver les coordonnées des points de rebroussement; car ces équations donnent tout de suite

$$
A=-3 C^{\frac{1}{3}}, \quad B=-3 C^{\frac{2}{2}},
$$

c'est-à-dire on a les équations

$$
\begin{aligned}
x^{2}+y^{2} & =a^{2}+b^{2}+k^{2}-3(a b k)^{\frac{2}{3}}, \\
b^{2} x^{2}+a^{2} y^{2} & =a^{2} b^{2}+\left(a^{2}+b^{2}\right) k^{2}-3(a b k)^{\frac{4}{3}}
\end{aligned}
$$

qui donnent les coordonnées des douze points de rebroussement.

Pour vérifier que ces points correspondent aux points de l'ellipse pour lesquels le rayon de courbure est égal à $k$; je prends $a \cos \theta, b \sin \theta$ pour les coordonnées d'un point sur l'ellipse, les coordonnées du centre de courbure seront données par

$$
a x=\left(a^{2}-b^{2}\right) \cos ^{3} \theta, \quad b y=-\left(a^{2}-b^{2}\right) \sin ^{3} \theta,
$$

et en supposant que le rayon de courbure soit égal à $k$, on a

$$
k=\frac{1}{a b}\left(a^{2} \sin ^{2} \theta+b^{2} \cos ^{2} \theta\right)^{\frac{3}{2}},
$$

et de là

Cela donne

$$
a^{2} \sin ^{2} \theta+b^{2} \cos ^{2} \theta=(a b k)^{\frac{2}{3}} .
$$

et de là on déduit

$$
\left(a^{2}-b^{2}\right) \cos ^{2} \theta=a^{2}-(a b k)^{\frac{2}{3}}, \quad-\left(a^{2}-b^{2}\right) \sin ^{2} \theta=b^{2}-(a b k)^{\frac{2}{3},}
$$

$$
\begin{aligned}
x^{2}+y^{2} & =\frac{1}{a^{2}-b^{2}}\left[\frac{1}{a^{2}}\left(a^{2}-(a b k)^{\frac{2}{3}}\right)^{3}-\frac{1}{b^{2}}\left(b^{2}-(a b k)^{\frac{2}{3}}\right)^{3}\right] \\
b^{2} x^{2}+a^{2} y^{2} & =\frac{1}{a^{2}-b^{2}}\left[\frac{b^{2}}{a^{2}}\left(a^{2}-(a b k)^{\frac{2}{3}}\right)^{3}-\frac{a^{2}}{b}\left(b^{2}-(a b k)^{\frac{2}{3}}\right)^{3}\right]
\end{aligned}
$$

valeurs qui s'accordent en effet avec les valeurs ci-dessus trouvées. 
La courbe parallèle à l'ellipse est de l'ordre 8 et il y a 8 points doubles, et 12 points de rebroussement; elle sera donc de la classe $56-16-36=4$. En effet pour mener d'un point donné une tangente à la courbe parallèle on peut décrire avec ce point comme centre un cercle rayon $k$, pour mener une tangente commune au cercle et à l'ellipse; la droite, menée par le point donné, parallèle à la tangente commune sera la tangente cherchée: on peut donc par un point donné mener 4 tangentes à la courbe, ou la courbe parallèle est de la classe 4. Au reste on peut trouver très facilement l'équation de la courbe parallèle en coordonnées tangentielles. Car en représentant par $\theta$ l'inclination d'une tangente quelconque de l'ellipse à l'axe de $x$, l'équation de cette tangente est

$$
x \cos \theta+y \sin \theta \quad-\sqrt{a^{2} \cos ^{2} \theta+b^{2} \sin ^{2} \theta}=0,
$$

et l'équation de la tangente parallèle de la courbe sera ainsi

$$
x \cos \theta+y \sin \theta-k-\sqrt{a^{2} \cos ^{2} \theta+b^{2} \sin ^{2} \theta}=0 ;
$$

donc en représentant cette équation par $\xi x+\eta y+\zeta=0$ on aura

et de là

$$
\xi: \eta: \zeta=\cos \theta: \sin \theta: k+\sqrt{a^{2} \cos ^{2} \theta+b^{2} \sin ^{2} \theta},
$$

$$
\zeta+k \sqrt{\xi^{2}+\eta^{2}}+\sqrt{a^{2} \xi^{2}+b^{2} \eta^{2}}=0,
$$

laquelle est l'équation dont il s'agit; la forme rationnelle est

$$
\left(a^{2}-k^{2}\right)^{2} \xi^{4}+\left(b^{2}-k^{2}\right)^{2} \eta^{4}+2\left(a^{2}-k^{2}\right)\left(b^{2}-k^{2}\right) \xi^{2} \eta^{2}-2\left(a^{2}+k^{2}\right) \zeta^{2} \xi^{2}-2\left(b^{2}+k^{2}\right) \zeta^{2} \eta^{2}+\zeta^{4}=0,
$$

équation du quatrième ordre comme cela devait être.

J'ai cru qu'il n'était pas nécessaire de faire voir comment on pourrait à moyen de l'équation tracer la courbe; en effet on trouve les différentes formes assez facilement par des considérations géométriques, en considérant la courbe comme la développée de l'évolute de l'ellipse: on se rend compte à ce moyen de ce qui est déjà dit par rapport aux points doubles et aux points de rebroussement.

Je remarque enfin que l'équation d'une normale quelconque de l'ellipse est

$$
a x \sin \theta-b y \cos \theta=\left(a^{2}-b^{2}\right) \sin \theta \cos \theta
$$

(où $\theta$ est un paramètre arbitraire): donc en considérant la trajectoire orthogonale de ce système de droites, on obtient

$$
(x d x+y d y) \sqrt{b^{2} d x^{2}+a^{2} d y^{2}}+\left(a^{2}-b^{2}\right) d x d y=0
$$

comme équation différentielle de la courbe parallèle à l'ellipse. L'intégrale de cette équation est donc l'équation du huitième ordre (contenant $k$ comme constante arbitraire) qu'on a ci-dessus trouvée.

Londres, 2 Stone Buildings, 22 Oct. 1860. 